\title{
Computation of Liquid-Vapor Critical Points for Multi-Component Mixtures
}

\author{
Wenlong Jia*, Changjun Li, Xia Wu \\ School of Petroleum Engineering, Southwest Petroleum University \\ CNPC Key Laboratory of Oil \& Gas Storage and Transportation, Southwest Petroleum University \\ Chengdu, Sichuan, China. \\ *Email: jiawenlong08@126.com
}

\begin{abstract}
Liquid-vapor critical point for a thermodynamic mixture is an important parameter for the design of mixture transportation and processing. The classical critical points model for the multicomponent mixture is based on the tangent plane distance in terms of the Helmholtz energy. In this work, we have applied the damped Newton-Raphson scheme to solve the model using the critical temperature, critical specific volume and change in mole fraction of each component as independent variables. According to numerical experiments, the convergence criteria, initial guesses of variables and damped factor formulation were determined. The method was tested on twenty five mixtures containing two to eleven components using the SRK and PR equation of state. Comparisons between the calculated critical parameters and experimental values illustrated the overall accuracy of the solution method is satisfactory. This method can solve all the variables simultaneously using the whole iteration, which is more reliable than the nested two-singe variable loops method proposed by Heidemann and Khalil. Thus it can be used for practical purposes of locating the liquid-vapor critical points of multi-component mixtures.
\end{abstract}

Keywords: Liquid-vapor critical points; multicomponent mixtures; equation of state; mathematic model; damped Newton-Raphson method.

\section{Introduction}

A critical state of a thermodynamic mixture is an equilibrium state that is essentially between regions of stability and instability with regard to the number of phases. A critical state is characterized by its critical point. At the critical point, liquid phase and the vapor phase have the same thermophysic prosperities such as viscosity, density, compressibility factor (Mokhatab et al, 2006). Locating the critical point is important for both hydrocarbon pipeline transmission and the oil reservoir development. For example, the critical point helps to determine the safety operating pressure and temperature range of liquid petroleum gas (LPG) or $\mathrm{CO}_{2}$ transmission pipeline ( $\mathrm{Li}$ et al, 2010). Also, knowledge of vapor-liquid critical is useful in determining the type of gas reservoir and whether retrograde condensation of evaporation is possible (Tian et al, 2003).

Normally, there are three ways which are experiments, empirical correlations and theoretical models to determine the critical points (Hicks et al, 1977). As the critical points obtained by experiments are expensive and the applicable range is limited, the critical points are frequently calculated by mathematical models.

Gibbs proposed the first rigorous thermodynamic definition of critical points for $\mathrm{n}$-component mixtures. The Gibbs criterion consists of two equations of partial composition derivatives of the Gibbs energy that must vanish at a critical point (Peng et al, 1977). Although the expressions are relatively simple for binary system, they become increasingly complex for the ternary and multicomponent system. Thus, the Gibbs criterion is less useful for practical calculations. To make the Gibbs criterion suitable for use, Heidemann and Khalil (Heidemann et al, 1980, Michelsen et al, 1988) expressed the partial derivatives of the molar Gibbs energy in terms of partial derivatives of the molar Helmholtz energy through transformation of the independent variables. Heidemann and Khalil also proposed the solution method for their model. The method employs two nested single-variable iteration loops using Newton-Raphson and it has been adopted by API Technical Databook (API, 2005). Wang (1999) used homotopy continuation technique for calculating critical points in binary mixtures. Benito et al. (2001) also used the Heidmann and Khalil criteria for critical points of binary and ternary system in conjunction with a numerical technique based on the interval Newton analysis method. Henderson et al. (2004) formulated the critical points as an optimization problem, which was solved by the simulated annealing algorithm. In their method, the independent variables are the pressure and temperature. Their methods also tested on several ternary systems. Luo et al. (2000) solved the Heidmann and Khalil's model using five-point numerical differentiation method. Justo-Garcia et al. (2008) applied the simulated annealing algorithm for the calculation of critical points of binary and multicomponent mixtures by using appropriate cooling schedule parameters to minimize the computer time for the solution of a critical point formulated as an optimization problem. Recently, Henderson et al. (2010) used differential evolution algorithms for the first time in the prediction of critical points of several multicomponent 
mixtures, but the critical points could be calculated in a few of hundredths of a second.

Sum of all, except for Justo-Garcia et al. (2008) and Henderson (2010), there are scarce literatures have applied their methods for the critical points calculation of multicomponent mixtures. However, lots of the hydrocarbon mixtures such as the condensed gas are consisted of more than seven components. Therefore, locating the liquid-vapor critical point is a problem worth studying.

The aim of this work is to research the liquid-vapor critical point calculation method for multicomponent mixutres. In Section 2, we introduced the critical points model developed by Heidemann, Khalil and Bentio. In Section 3, we applied the damped Newton-Raphson scheme to solve the model. In order to obtain the liquid-vapor critical point, the independent variables in the model, the iteration format, the convergence criteria, initial guesses of variables and damped factor were determined. Finally, the liquid-vapor critical points of twenty five mixtures from literatures are used to test the accuracy and robustness of the proposed solution method.

\section{Critical Points Model}

Gibbs presented a first critical point criterion which is in terms of the Gibbs free energy $G$. Heidemann and Khalil replaced the Gibbs free energy with Helmholtz free energy $A$. On the basis of a Taylor series expansion of $A$, and considering the second and third order terms, Heidemann, Khalil and Bentio et al formulated the critical condition for a mixture of $C$ components as follows (Heidemann et al, 1980, Benito et al. 2001,):

$$
\begin{aligned}
& \sum_{j=1}^{C} \sum_{i=1}^{C}\left(\frac{\partial\left(\ln f_{g i}\right)}{\partial n_{j}}\right)_{T, V} \Delta n_{i} \Delta n_{j}=0 \\
& \sum_{m=1}^{C} \sum_{j=1}^{C} \sum_{i=1}^{C}\left(\frac{\partial^{2}\left(\ln f_{g i}\right)}{\partial n_{j} \partial n_{m}}\right)_{T, v} \Delta n_{i} \Delta n_{j} \Delta n_{m}=0
\end{aligned}
$$

Where, $C$ is the total number of components, $n_{i}$ and $n_{j}$ are mole fraction of $i^{\text {th }}$ and $j^{\text {th }}$ components, $\Delta n_{i}, \Delta n_{j}, \Delta n_{m}$ are changes in mole fraction of $i^{\text {th }}, j^{\text {th }}$ and $m^{\text {th }}$ components. $f_{g i}$ is fugacity of $i^{\text {th }}$ component.

In equation (1) and equation (2), the fugacities are the most important parameters which are often derived from generalized cubic EoS. Equation (1) and equation (2) are transformed into the following equations using the Soave modification of the Redlich-Kwong (SRK) EoS and PengRobinson (PR) EoS.

$$
\begin{aligned}
& R T\left(\frac{\Delta n_{i}}{n_{i}}+F_{1}\left(\beta_{i} \bar{N}+\bar{\beta}\right)+\beta_{i} F_{1}^{2} \bar{\beta}\right)+ \\
& \frac{a}{b}\left(\beta_{i} \bar{\beta} F_{3}-\frac{F_{5}}{a} \sum_{j=1}^{c} a_{i j} \Delta n_{j}+F_{6}\left(\beta_{i} \bar{\beta}-\alpha_{i} \bar{\beta}-\bar{\alpha} \beta_{i}\right)\right)=0 \\
& R T\left(-\sum_{i=1}^{C} \frac{\Delta n_{i}^{3}}{n_{i}^{2}}+3 \bar{N}\left(\bar{\beta} F_{1}\right)^{2}+3\left(\bar{\beta} F_{1}\right)^{3}\right) \\
& +\frac{a}{b}\left(3 \bar{\beta}^{2}(2 \bar{\alpha}-\bar{\beta})\left(F_{2}+F_{3}+F_{6}\right)-2 \bar{\beta}^{3} F_{4}-3 \bar{\beta} \bar{a} F_{6}\right)=0
\end{aligned}
$$

Where, $i$ is the number of mixtures $(i=1,2, \cdots, C) . F_{1}, F_{2}$, $F_{3}, F_{4}, F_{5}, F_{6}, a, b, \beta_{i}, \bar{N}, \bar{\beta}, a_{i j}, \alpha_{i}, \bar{\alpha}$ are parameters related to the EoS.

There is an error in the paper Benito et al.(2001). In Benito's paper, the first term in the first bracket of equation (4) is $-\sum_{i=1}^{C} \Delta n_{i}^{3} / n_{i}^{2}$ which means the equation represents $\mathrm{C}$ equations. Obviously, the total number of the equations is differ with that of Eq.(2). This work corrected the error, and the correct term is $-\sum_{i=1}^{C}\left(\Delta n_{i}^{3} / n_{i}^{2}\right)$.

$$
\begin{aligned}
& \bar{N}=\sum_{i=1}^{C} \Delta n_{i} \\
& \alpha_{k}=\frac{\sum_{i=1}^{C} n_{i} a_{i k}}{a} \\
& \bar{\alpha}=\sum_{i=1}^{C} \Delta n_{i} \alpha_{i}
\end{aligned}
$$

$\bar{a}=\frac{1}{a} \sum_{i=1}^{C} \sum_{j=1}^{C} \Delta n_{i} \Delta n_{j} a_{i j}$

$\beta_{i}=\frac{b_{i}}{b}$

$$
\bar{\beta}=\sum_{i=1}^{C} \Delta n_{i} \beta_{i}
$$

$F_{1}=\frac{1}{K-1}$

$$
F_{2}=\frac{2}{D_{1}-D_{2}}\left[\frac{D_{1}}{K+D_{1}}-\frac{D_{2}}{K+D_{2}}\right]
$$

$$
F_{3}=\frac{2}{D_{1}-D_{2}}\left[\left(\frac{D_{1}}{K+D_{1}}\right)^{2}-\left(\frac{D_{2}}{K+D_{2}}\right)^{2}\right]
$$

$F_{4}=\frac{2}{D_{1}-D_{2}}\left[\left(\frac{D_{1}}{K+D_{1}}\right)^{3}-\left(\frac{D_{2}}{K+D_{2}}\right)^{3}\right]$

$F_{5}=\frac{2}{D_{1}-D_{2}} \ln \left(\frac{K+D_{1}}{K+D_{2}}\right)$

$F_{6}=\frac{2}{D_{1}-D_{2}}\left[\left(\frac{D_{1}}{K+D_{1}}-\frac{D_{2}}{K+D_{2}}\right)-\ln \left(\frac{K+D_{1}}{K+D_{2}}\right)\right]$

$K=\frac{V_{c}}{n b}=\frac{v_{c}}{b}$

$a=\sum_{i=1}^{C} \sum_{j=1}^{C}\left(\frac{n_{i} n_{j}}{n^{2}}\right) a_{i j}$ 
$b=\sum_{i=1}^{c}\left(\frac{n_{i}}{n}\right) b_{i}$

$a_{i j}=\left(a_{i} a_{j}\right)^{0.5}\left(1-k_{i j}\right)$

$a_{i}=\frac{\left(R T_{c i}\right)^{2} \eta}{P_{c i}}\left[1+c_{i}\left(1-\left(\frac{T_{c}}{T_{c i}}\right)^{0.5}\right)\right]^{2}$

$D_{1}=\frac{u_{0}+\sqrt{u_{0}^{2}-4 w_{0}}}{2}$

$D_{2}=\frac{u_{0}-\sqrt{u_{0}^{2}-4 w_{0}}}{2}$

For SRK EoS:

$\eta=0.42748 \quad, \quad c_{i}=0.48+1.574 w_{i}-0.176 w_{i}^{2}$,

$b_{i}=0.08664 R T_{c i} / P_{c i}, u_{0}=1, w_{0}=0$.

\section{For PR EoS}

$\eta=0.45724 \quad, \quad c_{i}=0.37464+1.54226 w_{i}-0.26992 w_{i}^{2}$,

$b_{i}=0.07780 R T_{c i} / P_{c i}, u_{0}=2, w_{0}=-1$.

Where $T_{c}$ is the critical temperature of the mixture, $v_{c}$ is the critical specific volume of the mixture, $T_{c i}$ is the critical temperature of $i^{\text {th }}$ component, $P_{c i}$ is the critical pressure of $i^{\text {th }}$ component, $w_{i}$ is the acentric factor of $i^{\text {th }}$ component, $k_{i j}$ is the binary interaction coefficient. These parameters can be found in API Databook (API, 2005) or some commercial software such as Aspen HYSYS and SIMSCI PIPEPHASE.

Set $\Delta \mathbf{n}=\left[\Delta n_{1}, \Delta n_{2}, \cdots, \Delta n_{C}\right]^{T}$. To ensure that it is nonzero, the vector $\Delta \mathbf{n}$ is normalized.

$\Delta \mathbf{n}^{T} \Delta \mathbf{n}-1=0$

Equations (3) (4) and (24) construct the critical points model of defined mixtures. Equation (3) should apply to each component, thus the model represents a system of $C+2$ equations with the $C+2$ variables $T_{c}, v_{c}$ and $\Delta n_{i}(i=1,2, \cdots$, $C)$. The critical temperature $T_{c}$, critical specific volume $v_{c}$ and $\Delta \mathbf{n}$ can be gained by solving the equations. The critical pressure $P_{\mathrm{c}}$ can then be computed directly from the pressure-explicit EoS.

\section{Model Solution}

\subsection{Damped Newton-Raphson Iteration Scheme}

In order to calculate the critical point of a mixture, the above equations should be satisfied simultaneously. Heidemann and Khalil employed two nested single-variable iteration loops and the Newton iteration method to solve the model. The inner loop is holding $v_{c}$ constant, and iterates $T_{c}$ to find the equation (3) is equal to zero using a Newton iteration scheme. The outer loop is calculating the values of $\Delta n_{i}$ and checking whether equation (4) is satisfied on the basis of $T_{c}$ obtained from the inner loop. If it is satisfied, exit the iteration. Otherwise, repeat the inner loop and obtain the new values of $T_{c}$ using Newton iteration scheme until equation (4) is satisfied (Michelsen et al, 1988; API,
2005).

This work replaced the two nested single-variable iteration with a whole iteration using Newton-Raphson iteration scheme. However, we found that if the total number of components is more than five and traditional Newton-Raphson method is employed to solve the critical points model, the Newton-Naphson method may not converge successfully. To overcome the problem, this work used the damped Newton-Naphson (Abbasbandy et al, 2003; Crisfield et al, 1984). Different with the traditional Newton iteration method, the convergence of damped NewtonNaphson is not only dependent on the initial guesses of variables, but also relates to the damped factor.

Nominate the equations ( 3 ) is $f_{11}-f_{l c}$, equation (4) is $f_{2}$ and equation (24) is $f_{3}$. Based on Taylor series expansion of the $C+2$ equations, and considering the first order terms, the iteration format can be built as follows:

Where, $\Delta T_{c}, \Delta v_{c}, \Delta \Delta n_{i}$ are the correction terms of the critical temperature, critical specific volume of the mixture and change in mole fraction of $i^{\text {th }}$ component respectively. $x(k)$ is the variables set of the $k^{\text {th }}$ iteration time, $x^{(k)}=\left(T^{(k)}, v^{(k)}, \Delta n_{1}^{(k)}, \cdots, \Delta n_{c}^{(k)}\right)$.

To ensure the results calculated are the vapor-liquid critical points, based on the method introduced in API Data Book and numerical experiments, we proposed the initial values of the variables can be set as follows.

Initial value of the critical specific volume:

$v_{c}=\sum_{i=1}^{C} n_{i} v_{c i}$

Initial value of the critical temperature:

$T_{c}=3 \sum_{i=1}^{C} n_{i} T_{c i}$

Initial values of the change in mole fraction of each component:

$$
\Delta n_{i}=n_{i}^{2 / 3}
$$

Where, $v_{c i}$ is the critical specific volume of $i^{\text {th }}$ component. 
To ensure the iteration is convergent, the damped factor is calculated by equation (29).

$$
x^{k+1}=x^{k}+\frac{1}{1+Q \exp (-0.5 k)} \Delta x
$$

If $Q=0$, the damped Newton-Raphson scheme is entirely equivalent to traditional Newton-Raphson scheme.

Considering there are magnitude difference between the temperature and the other variables, the different convergence criterion for each variable are proposed as follows:

$|\Delta T|<0.2$

$|\Delta v|<0.01$

$\left|\Delta \Delta n_{i}\right|<0.01 i=1,2, \cdots, C$

When above convergence criteria are satisfied simultaneously, exit the iteration and output the results.

\subsection{Determining the damped factor}

In equation (29) the damped factor is dependent on $Q$ which can be obtained by numerical experiments. We take a condensate gas which contains eleven components as a test sample. For different $Q$, the convergent curves of $T_{c}$ and $v_{c}$ are depicted in Figure 1 and Figure 2.

Table 1. Molar composition of the tested condensate gas.

\begin{tabular}{llll}
\hline Component & Mole fraction & Component & Mole fraction \\
\hline $\mathrm{CO}_{2}$ & 0.0150 & $\mathrm{nC}_{4} \mathrm{H}_{10}$ & 0.0329 \\
$\mathrm{~N}_{2}$ & 0.0033 & $\mathrm{iC}_{5} \mathrm{H}_{12}$ & 0.0402 \\
$\mathrm{CH}_{4}$ & 0.5205 & $\mathrm{nC}_{5} \mathrm{H}_{12}$ & 0.0458 \\
$\mathrm{C}_{2} \mathrm{H}_{6}$ & 0.0823 & $\mathrm{C}_{6} \mathrm{H}_{14}$ & 0.0503 \\
$\mathrm{C}_{3} \mathrm{H}_{8}$ & 0.0633 & $\mathrm{C}_{7} \mathrm{H}_{16}+$ & 0.1162 \\
$\mathrm{iC}_{4} \mathrm{H}_{10}$ & 0.0302 & & \\
\hline
\end{tabular}

As depicted in Figure 1, the temperature cannot converge to a certain value if $Q=0$ (traditional NewtonRaphson scheme) and $Q=400$. However, the desired temperature can be obtained when $Q$ is equal to or larger than 500. The similar phenomena also can be seen in Figure 2. Also, the critical specific volume cannot converge to a correct value if $Q=0$ or $Q=400$, while it converge to the same and correct value when $Q=500$ and $Q=600$. Then, we can give the correct liquid-vapor critical temperature is $407.6 \mathrm{~K}$, critical specific volume is $1.523 \mathrm{~m}^{3} / \mathrm{kmol}$, the critical pressure is $13706 \mathrm{kPa}$, corresponding changes in mole fraction of all components are $0.0055,-0.0056$, $0.1936,0.1100,0.1650,0.1065,0.1271,0.1926,0.2310$, $0.3102,0.8420$.

Note that the total iteration time is 35 when $Q=500$ and total iteration time is 36 when $Q=600$. That can be explained that the step length of each variable modification is decreasing with the increasing of $Q$. Finally, we choose 500 as the optimal value of $Q$. Then equation (29) is expressed as:

$$
x^{k+1}=x^{k}+\frac{1}{1+500 \exp (-0.5 k)} \Delta x
$$

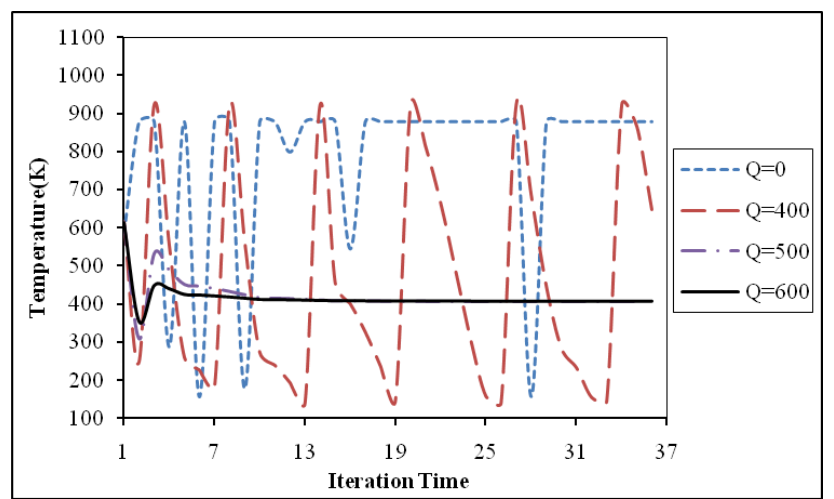

Figure 1. The critical temperature convegent curves.

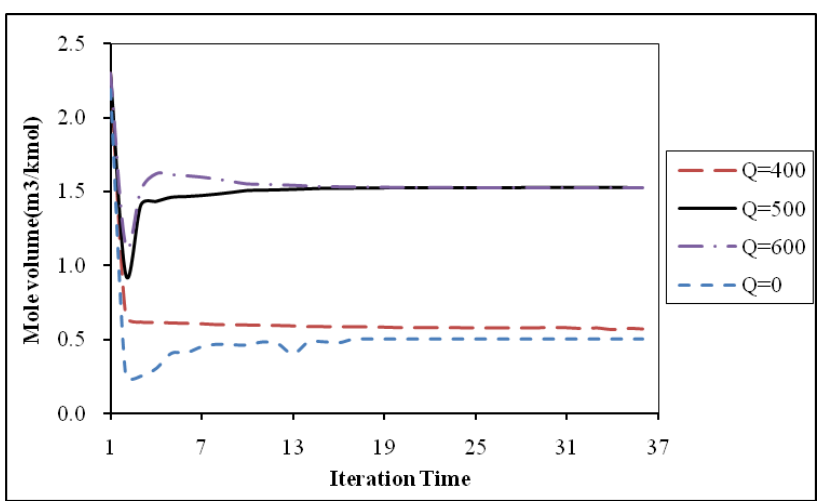

Figure 2. The critical specific volume convegent curves.

The choice of the initial values, convergence criteria and damped factor is a very important aspect in the implementation of the solution method since it strongly affects the robustness of the method. The proposed initial values, convergence criterions and damped factor make the iteration method robust for locating critical points of multicomponent mixtures up to 11 components.

\section{Results and Discussion}

The solution procedures for locating the liquid-vapor critical point involve a large amount of matrixes calculation. In order to test the procedures presented, the computer program was developed using Microsoft Visual Basic and MATLAB hybrid programming technical (Li et al, 2010).

In this example, critical temperatures and pressures have been calculated for a total for twenty five mixtures which were gained from 11 literatures. This compositions for all the mixtures studied are given in Table 2. This table includes two two-component mixtures, eight threecomponent mixtures, four four-component mixtures, three five-component mixtures, one six-component mixture, one seven-component mixture, one ten-component mixture and four eleven-component mixtures, containing from methane to n-heptane, $\mathrm{CO}_{2}, \mathrm{~N}_{2}$ were used to test the solution program.

The basic parameters, such as binary interaction parameters, molar weight, acentric factor and critical parameters of each component are obtained from the software named Aspen HYSYS 2006.

Table 3 presents the calculated critical pressures and temperatures for the twenty-five mixtures given in Table 2 using PR EoS and SRK EoS. To analyze the accuracy of the solution method, two kinds of error evaluation methods are defined. The first one is relative error (RE) defined by equation (34). 
Table 2. Compositions of multicomponent systems studied.

\begin{tabular}{|c|c|c|c|c|c|c|c|c|c|c|c|}
\hline \multirow{2}{*}{$\begin{array}{l}\text { Mixture } \\
\text { no. }\end{array}$} & \multicolumn{11}{|c|}{ Mixture composition (mole fraction) } \\
\hline & $\mathrm{CO}_{2}$ & $\mathrm{~N}_{2}$ & $\mathrm{CH}_{4}$ & $\mathrm{C}_{2} \mathrm{H}_{6}$ & $\mathrm{C}_{3} \mathrm{H}_{8}$ & $\mathrm{iC}_{4} \mathrm{H}_{10}$ & $\mathrm{nC}_{4} \mathrm{H}_{10}$ & $\mathrm{iC}_{5} \mathrm{H}_{12}$ & $\mathrm{nC}_{5} \mathrm{H}_{12}$ & $\mathrm{C}_{6} \mathrm{H}_{14}$ & $\mathrm{C}_{7} \mathrm{H}_{16^{+}}+$ \\
\hline 1 & & & 0.1000 & 0.9000 & & & & & & & \\
\hline 2 & 0.9500 & 0.0500 & & & & & & & & & \\
\hline 3 & & 0.0700 & 0.4600 & & & & & & & 0.4700 & \\
\hline 4 & & & & 0.4290 & & & 0.3730 & & & & 0.1980 \\
\hline 5 & & & & 0.7260 & & & 0.1710 & & & & 0.1030 \\
\hline 6 & & & & 0.5140 & & & 0.4120 & & & & 0.0740 \\
\hline 7 & & & & 0.8010 & & & & 0.0640 & & & 0.1350 \\
\hline 8 & & & & 0.6120 & & & & 0.2710 & & & 0.1170 \\
\hline 9 & & & & 0.6150 & & & & 0.2960 & & & 0.0890 \\
\hline 10 & & 0.0465 & 0.4530 & & 0.5005 & & & & & & \\
\hline 11 & & 0.0490 & 0.4345 & 0.0835 & 0.4330 & & & & & & \\
\hline 12 & & & & 0.6168 & & & 0.1376 & 0.0726 & & & 0.1730 \\
\hline 13 & & & & 0.2542 & 0.2547 & & 0.2554 & & 0.2357 & & \\
\hline 14 & & & & & 0.4858 & & 0.3316 & & 0.1213 & 0.0613 & \\
\hline 15 & & & 0.2019 & 0.2029 & 0.2033 & & 0.2038 & & 0.1881 & & \\
\hline 16 & & & 0.7057 & 0.0669 & 0.0413 & & 0.0508 & & 0.1353 & & \\
\hline 17 & & & 0.6626 & 0.1093 & 0.1057 & & & & 0.0616 & 0.0608 & \\
\hline 18 & & & 0.1015 & 0.3573 & 0.2629 & & 0.1794 & & 0.0657 & 0.0332 & \\
\hline 19 & & 0.0140 & 0.9430 & 0.0270 & 0.0074 & & 0.0049 & & 0.0010 & 0.0027 & \\
\hline 20 & 0.0120 & & 0.9089 & 0.0440 & 0.0191 & 0.0033 & 0.0060 & 0.0021 & 0.0013 & 0.0015 & 0.0018 \\
\hline 21 & 0.0109 & 0.0884 & 0.8286 & 0.0401 & 0.0174 & 0.0030 & 0.0055 & 0.0019 & 0.0012 & 0.0014 & 0.0006 \\
\hline 22 & 0.0091 & 0.2441 & 0.6870 & 0.0333 & 0.0144 & 0.0030 & 0.0040 & 0.0016 & 0.0010 & 0.0011 & 0.0014 \\
\hline 23 & 0.0020 & 0.2400 & 0.7364 & 0.0120 & 0.0053 & 0.0010 & 0.0015 & 0.0005 & 0.0004 & 0.0004 & 0.0005 \\
\hline 24 & 0.0030 & 0.1130 & 0.8580 & 0.0150 & 0.0060 & 0.0012 & 0.0018 & 0.0006 & 0.0004 & 0.0004 & 0.0006 \\
\hline 25 & 0.0100 & 0.1611 & 0.7625 & 0.0369 & 0.0160 & 0.0028 & 0.0051 & 0.0018 & 0.0011 & 0.0012 & 0.0015 \\
\hline
\end{tabular}

Table 3. Critical parameters obtained from calculation and experiments.

\begin{tabular}{|c|c|c|c|c|c|c|c|c|}
\hline \multirow{2}{*}{$\begin{array}{c}\text { Mixture } \\
\text { no. }\end{array}$} & \multirow{2}{*}{$\begin{array}{l}\text { Number. of } \\
\text { components }\end{array}$} & \multicolumn{2}{|c|}{ Experimental } & \multicolumn{2}{|c|}{ SRK EOS } & \multicolumn{2}{|c|}{ PR EOS } & \multirow{2}{*}{$\begin{array}{c}\text { Data } \\
\text { Source }\end{array}$} \\
\hline & & $T_{c}(\mathrm{~K})$ & $P_{c}(\mathrm{kPa})$ & $T_{c}(\mathrm{~K})$ & $P_{c}(\mathrm{kPa})$ & $T_{c}(\mathrm{~K})$ & $\begin{array}{c}P_{c}(\mathrm{kPa} \\
)\end{array}$ & \\
\hline 1 & 2 & 299 & 5322 & 295 & 5235 & 299 & 5328 & (API, 2005) \\
\hline 3 & 3 & 462 & 1170 & 463 & 1168 & 459 & 1178 & (Benito,2001) \\
\hline 4 & 3 & 438 & 6612 & 442 & 6375 & 439 & 6320 & (Brunner,1990) \\
\hline 5 & 3 & 386 & 7605 & 390 & 7591 & 388 & 7462 & (Brunner,1990) \\
\hline 6 & 3 & 400 & 6405 & 405 & 6298 & 404 & 6224 & (Brunner,1990) \\
\hline 7 & 3 & 391 & 8101 & 396 & 8416 & 392 & 8260 & (Brunner,1990) \\
\hline 8 & 3 & 421 & 7156 & 423 & 7085 & 420 & 7009 & (Brunner,1990) \\
\hline 9 & 3 & 416 & 7060 & 417 & 6947 & 414 & 6879 & (Brunner,1990) \\
\hline 10 & 3 & 313 & 9232 & 324 & 9205 & 321 & 9141 & (Yarborough,1970) \\
\hline 11 & 4 & 313 & 8963 & 318 & 9093 & 316 & 9028 & (Yarborough,1970) \\
\hline 12 & 4 & 423 & 7412 & 426 & 7477 & 423 & 7389 & (Ekiner,1965) \\
\hline 13 & 4 & 406 & 5113 & 411 & 5095 & 409 & 5037 & (Etter,1961) \\
\hline 14 & 4 & 418 & 4506 & 420 & 4443 & 419 & 4410 & (Etter,1961) \\
\hline 16 & 5 & 308 & 13700 & 320 & 14884 & 316 & 14499 & (Hanson, 1945) \\
\hline 17 & 5 & 310 & 13748 & 324 & 14706 & 318 & 14443 & (Hanson, 1945) \\
\hline 18 & 6 & 376 & 6536 & 382 & 6508 & 380 & 6456 & (Etter,1961) \\
\hline 19 & 7 & 201 & 5578 & 202 & 5938 & 202 & 5906 & (Gonzalez,1968) \\
\hline 20 & 10 & 211 & 6378 & 211 & 6635 & 211 & 6602 & (Gore, 1952) \\
\hline 21 & 11 & 204 & 6584 & 204 & 6982 & 204 & 6704 & (Davis, 1954) \\
\hline 22 & 11 & 189 & 6708 & 180 & 6112 & 181 & 6238 & (Davis, 1954) \\
\hline 23 & 11 & 183 & 5618 & 184 & 6081 & 183 & 6108 & (Davis, 1954) \\
\hline 24 & 11 & 193 & 5829 & 193 & 5937 & 193 & 5916 & (Davis, 1954) \\
\hline 25 & 11 & 198 & 6674 & 192 & 6573 & 194 & 6878 & (Davis, 1954) \\
\hline
\end{tabular}

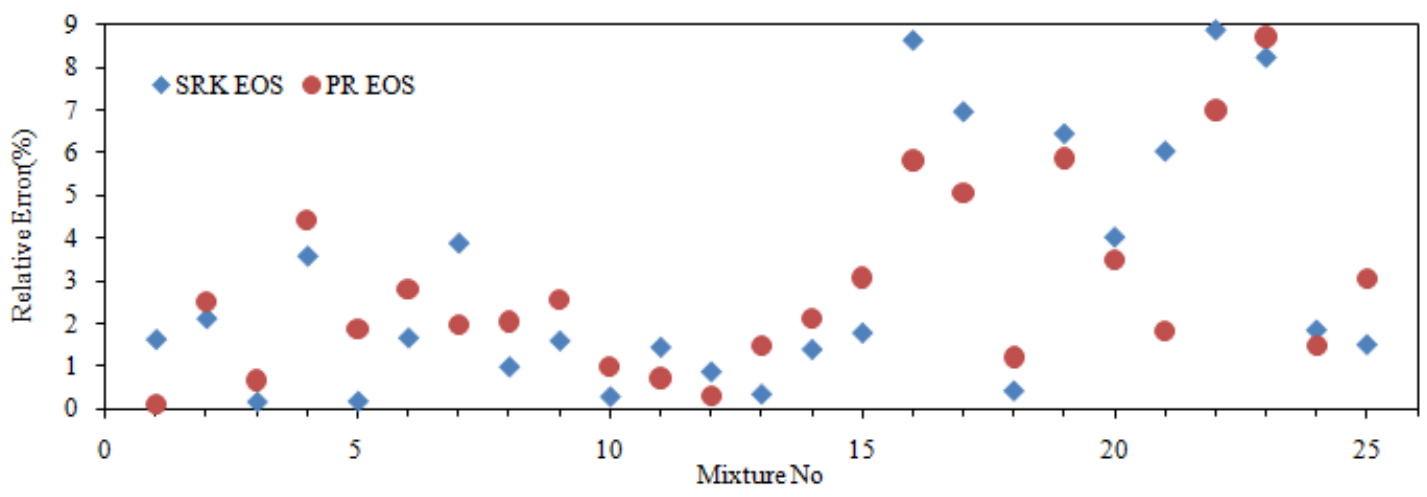

Figure 3. Relative errors for critical pressures 


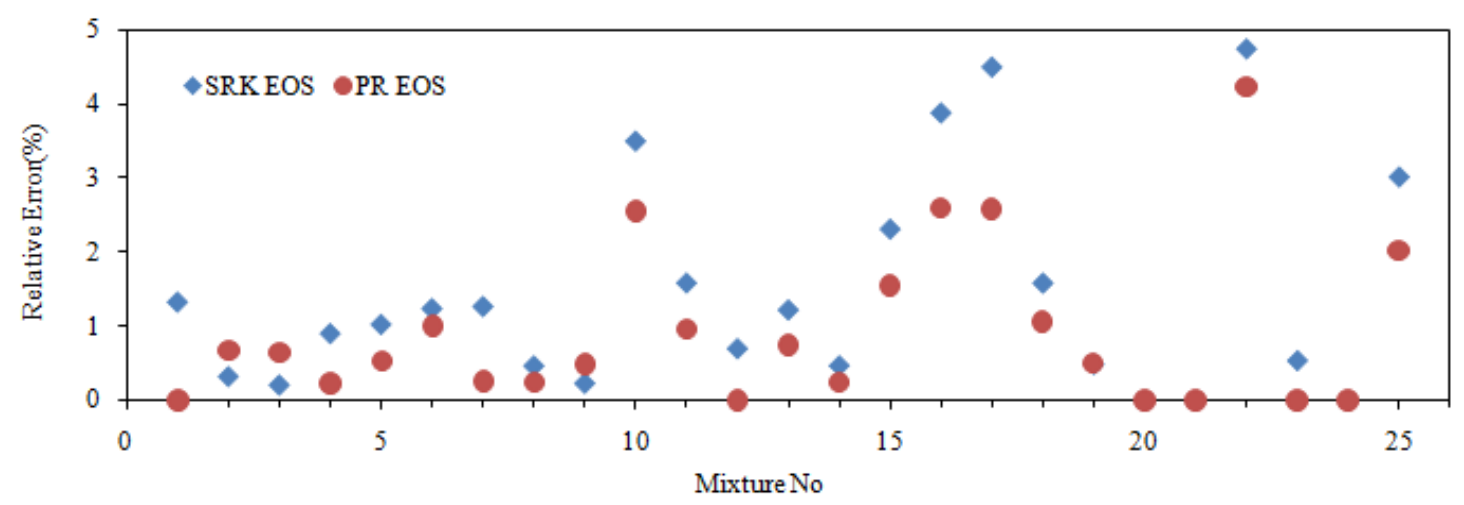

Figure 4. Relative errors for critical temperatures

$R E=\frac{\left|x_{c a l}-x_{\text {exp }}\right|}{x_{\exp }} \times 100 \%$

Where, $x_{c a l}$ represents the calculated critical temperature or pressure, $x_{\exp }$ represents the critical temperature or pressure from experiments.

The relative errors between the experimental and calculated values are depicted in Figure 3 and Figure 4. The average relative error of critical pressures calculated using SRK EoS is $3.00 \%$, while that calculated using PR EoS is $2.85 \%$. The average relative error of critical temperatures calculated using SRK EoS is $1.43 \%$, and the corresponding error of PR EoS is $0.92 \%$. That means accuracy of the critical temperatures prediction is better than that of the critical pressures prediction. The overall critical parameters prediction accuracy of PR EoS is better than SRK EoS.

The second one is average absolute deviation $(A A D)$ defined by equation (35).

$$
A A D=\frac{1}{C} \sum_{i=1}^{N} \max \left|x_{c a l}-x_{\text {exp }}\right|
$$

The $A A D$ of critical temperatures calculated using SRK EoS is $4.44 \mathrm{~K}$, and that calculated using PR EoS is $2.80 \mathrm{~K}$. The $A A D$ of critical pressures calculated using SRK EoS is $240.28 \mathrm{kPa}$, and that calculated using PR EoS is $209.40 \mathrm{kPa}$. The overall accuracy of the solution method is slightly better than the simulated annealing technique reported by Justo-Garcia et al.(2008).

\section{Conclusions}

Based on the damped Newton-Raphson scheme, this paper researched the solution method of the critical model proposed by Hidemann and Khalil and developed by Stradi et al. This method used the critical temperature, critical specific volume and mole change in mole fraction of each component as independent variables. All the variables can be solved simultaneously through a whole iteration, which is more reliable than the nested two-singe variable loops method. Furthermore, the initial guesses of variables, convergence criteria of the iteration, and the damped factor were determined by using of numerical experiments. the choice of the values makes the iteration method robust for locating critical points of multicomponent mixtures.

The solution method was tested successfully on 25 mixtures which contain hydrocarbons from methane to nheptane, $\mathrm{CO}_{2}, \mathrm{~N}_{2}$. The analysis of errors presented the overall accuracy of the method is satisfactory. The method provides a practical way to locating the liquid-vapor critical point of the multicomponent mixtures.

\section{Nomenclature}

$C \quad$ total number of components

$n \quad$ mole fraction of component

$\Delta n \quad$ change in mole fraction

$T \quad$ temperature

$v \quad$ critical specific volume,

$f_{g} \quad$ fugacity

$T_{c} \quad$ critical temperature of the mixture

$v_{c} \quad$ critical specific volume of the mixture

$P_{\mathrm{c}} \quad$ critical pressure of the mixture

$T_{c i} \quad$ critical temperature of $i^{\text {th }}$ component

$P_{c i} \quad$ critical pressure of $i^{\text {th }}$ component

$v_{c i} \quad$ critical volume of $i^{\text {th }}$ component

$w \quad$ accentric factor

$k_{i j} \quad$ binary interaction coefficient.

$\Delta T \quad$ correction term of the critical temperature

$\Delta v \quad$ correction term of the critical specific volume

$\Delta \Delta n \quad$ correction term change in mole fraction

$Q \quad$ parameter in damped Newton iteration method

$F_{1}, F_{2}, F_{3}, F_{4}, F_{5}, F_{6}, a, b, \beta_{i}, \bar{N}, \bar{\beta}, a_{i j}, \alpha_{i}, \bar{\alpha}$ parameters related with equation of state.

\section{Subscripts}

$i \quad i^{\text {th }}$ component

$j \quad j^{\text {th }}$ component

$m \quad m^{\text {th }}$ component

c critical condition

\section{Superscripts}

$k \quad k^{\text {th }}$ iteration step

\section{Acknowledgements}

This paper is a project Experiment and Theory Research of the Dynamic Characteristics of Gas Pipe Bridge in Pigging supported by National Natural Science Foundation of China (No. 51174172) and sub-project of National science and technology major project of China (No.2011ZX05054, No. 2011ZX05026-001-07).

\section{References}

Abbasbandy, S. (2003). Improving Newton-Raphson method for nonlinear equations by modified Adomian decomposition method. Applied Mathematics and Computation, 25, 887-893. 
API. (2005). Americal petroleum insititute Data Book,7th Edition, EPCON International and API, Houston, USA

Benito, A. S., Joan, F. B. and James, P. K. (2001). Reliable Computation of Mixture Critical Points", AIChE Journal, 47, 212-221.

Brunner, E. (1990). Fluid mixtures at high pressures. IX. Phase separation and critical phenomena in 23 (n-alkane +water) mixtures", Journal of Chemical Thermodynamics, 22 , 335-353.

Crisfield, M. A. (1984). Accelerating and damping the modified Newton-Raphson method, Computers \& Structures, 18,395-407.

Davis, P.C., Bertuzzi, A.F. and Gore, T.L. (1954). The phase and volumetricbehavior of natural gases at low temperatures and high pressures. Petroleum Transaction, AIME, 201, 245-250.

Etter, D.O., and Kay, W. B.(1961). Critical properties of mixtures of normal paraffin hydrocarbons, Journal of Chemical \& Engineering Data, 6, 409-413.

Ekiner, O. (1965). Critical Temperature and Pressure for Multicomponet Hydrocarbon Systems. Ph.D. Dissertation, Northwestern University, Evanston, IL, 1965.

Gonzalez, M. H., and Lee, A. L.(1968). Dew and bubble points of simulated natural gases. Journal of Chemical \& Engineering Data ,13,172-176.

Gore, T. L., Davis, P. C. and Kurata, F.(1952). Phase and volumetric behavior of natural gases at low temperatures. Petroleum Transaction, AIME, 279-284

Hanson, G.H., and Brown, C.G. (1945). Vapor-liquid equilibria in mixtures of volatile paraffins. Industrial Engineering Chemistry, 37, 821-825.

Heidemann, R. A., and Khalil, A.M. (1980). The Calculation of Critical points. AIChE Journal, 26, 769779.

Henderson, N., Freitas, L. and Platt, G. M.(2004). Prediction of critical points: A new methodology using global optimization. AIChE Journal. 50,1300-1314.

Henderson, N., Wagner, F. S. and Nelza E. B.(2010). Calculation of Critical Points of Thermodynamic Mixtures with Differential Evolution Algorithms. Ind. Eng. Chem. Res. 49,1872-1882.

Hicks, C. P., and Young, C. L. (1977). Theoretical Prediction of Phase Behavior at High Temperatures and Pressures for Non-Polar Mixtures: I. Computer Solution Techniques and Stability Tests. Journal of the Chemical Society, Faraday Transactions 2: Molecular and Chemical Physics, 73, 597-612
Justo-Garcia, D. N., and Garcia-Sanchez, F. (2008).

Application of the simulated annealing technique to the calculation of critical points of multicomponent mixtures with cubic equations of state. Fluid Phase Equailibria, 264, 164-173.

Li, C. J. Wu, X. (2010). Study on Operating Parameters of $\mathrm{CO}_{2}$ Supercritical Pipelines. Proceedings of International Oil and Gas Conference and Exhibition in China, Beijing, China, SPE 131424

Li, C. J., Wu, X. and Jia, W. L.(2010). Research of heated oil pipeline shutdown and restart process based on VB and MATLAB. International Journal of Education and Computer Science, 2, 18-24.

Luo, K., Song W. J. and Fang, Y. S.(2000). Critical point Calculation of gas condensate fluid. Natural Gas Industry, 20, 65-67.

Mokhatab, S. Poe, W. A. (2006). Handbook of Natural Gas Transmission and Processing. Gulf Professional Publishing, Burlington, USA.

Michelsen, M. L., and Heidemann, R. A.(1988). Calculation of tri-critical points", Fluid Phase Equilibria, 39, 5374.

Peng, D. Y., and Robinson, D. B. (1997). A rigorous method for predicting the critical the critical prosperities of multicomponent system from equation of state. AIChE Journal, 23,137-144.

Seevam, P. J., and Race, M. (2007). The Impact of Impurities on Supercritical $\mathrm{CO}_{2}$ Pipeline Transport for Sequestration. Proceedings of the First International Forum on Petreolum for Ph. D Candidates, Beijing, China, 2915-2927

Tian, C. B., Luo, K. and Hu, Y. L. (2003). Determination method and affecting factors for dew point of condensate gas. Acta Petrolei Sinica, 24,73-76

Wang, M. C., Wong D. S. and Chen, H.(1999). Homotopy continuation method for calculating critical loci of binary mixtures. Chemical Engineering Science, 54, 3873-3883.

Yarborough, L., and Smith, R. L. (1970). Solvent and driving gas compositions for miscible slug displacement. SPE Journal, 10, 298-305.

Appendix:The Derivation of Equations (3) and (4) The general form of SRK EoS and PR EoS is

$$
P=\frac{R T}{v-b}-\frac{a}{\left(v+b D_{1}\right)\left(v+b D_{2}\right)}
$$

The parameters in the equation are listed in the paper. Based on the EoSs, the fugacity of component $i$ can be written as follows:

$$
\ln \left(f_{g i}\right)=R T\left[\ln \left(n_{i}\right)+\frac{b_{i}}{v-b}\right]+\frac{a b_{i}}{b}\left[\frac{1}{b\left(D_{1}-D_{2}\right)}\left(\ln \left(\frac{v+b D_{1}}{v+b D_{2}}\right)-\left(\frac{b D_{1}}{v+b D_{1}}-\frac{b D_{2}}{v+b D_{2}}\right)\right)\right]-\frac{\partial a}{\partial n_{i}} \frac{1}{b\left(D_{1}-D_{2}\right)} \ln \left(\frac{v+b D_{1}}{v+b D_{2}}\right)
$$

The derivates in the quadratic form are: 


$$
\begin{aligned}
\left(\frac{\partial \ln f_{i}}{\partial n_{j}}\right)= & R T\left[\frac{\delta_{i j}}{n_{j}}+\frac{\left(b_{i}+b_{j}\right)}{v-b}+\frac{\left(b_{i} b_{j}\right)}{(v-b)^{2}}\right]+\frac{a b_{i} b_{j}}{b^{2}} \frac{1}{b\left(D_{1}-D_{2}\right)}\left[\left(\frac{b D_{1}}{v+b D_{1}}\right)^{2}-\left(\frac{b D_{2}}{v+b D_{2}}\right)^{2}\right] \\
& +\frac{2 a b_{i} b_{j}-b\left(\frac{\partial a}{\partial n_{i}} b_{j}+\frac{\partial a}{\partial n_{j}} b_{i}\right)}{b} \frac{1}{b\left(D_{1}-D_{2}\right)}\left[\frac{b D_{1}}{v+b D_{1}}-\frac{b D_{2}}{v+b D_{2}}\right]+\frac{-2 a b_{i} b_{j}+b\left(\frac{\partial a}{\partial n_{i}} b_{j}+\frac{\partial a}{\partial n_{j}} b_{i}\right)-2 a_{i j} b^{2}}{b^{3}} \ln \left(\frac{v+b D_{1}}{v+b D_{2}}\right)
\end{aligned}
$$

The terms in the cubic form are

$$
\begin{aligned}
\left(\frac{\partial^{2} \ln f_{i}}{\partial n_{j} \partial n_{m}}\right)= & R T\left[\frac{\delta_{i j m}}{n_{i}^{2}}+\frac{\left(b_{i} b_{j}+b_{j} b_{k}+b_{m} b_{i}\right)}{(v-b)^{2}}+\frac{2 b_{i} b_{j} b_{m}}{(v-b)^{3}}\right]-\frac{2 a b_{i} b_{j} b_{m}}{b^{3}} \frac{1}{b\left(D_{1}-D_{2}\right)}\left[\left(\frac{b D_{1}}{v+b D_{1}}\right)^{3}-\left(\frac{b D_{2}}{v+b D_{2}}\right)^{3}\right] \\
& +\frac{b\left(\frac{\partial a}{\partial n_{i}} b_{j} b_{m}+\frac{\partial a}{\partial n_{j}} b_{i} b_{m}+\frac{\partial a}{\partial n_{m}} b_{i} b_{j}\right)-3 a b_{i} b_{j} b_{m}}{b^{3}} \frac{2}{b\left(D_{1}-D_{2}\right)}\left[\left(\frac{b D_{1}}{v+b D_{1}}\right)^{2}-\left(\frac{b D_{2}}{v+b D_{2}}\right)^{2}\right] \\
& +\frac{2\left[b\left(\frac{\partial a}{\partial n_{i}} b_{j} b_{m}+\frac{\partial a}{\partial n_{j}} b_{i} b_{m}+\frac{\partial a}{\partial n_{m}} b_{i} b_{j}\right)-3 a b_{i} b_{j} b_{m}\right]-2 b^{2}\left(a_{i j} b_{m}+a_{i m} b_{j}+a_{j m} b_{i}\right)}{b^{3}} \frac{1}{b\left(D_{1}-D_{2}\right)}\left[\frac{b D_{1}}{v+b D_{1}}-\frac{b D_{2}}{v+b D_{2}}\right] \\
& +\frac{2\left[b\left(\frac{\partial a}{\partial n_{i}} b_{j} b_{m}+\frac{\partial a}{\partial n_{j}} b_{i} b_{m}+\frac{\partial a}{\partial n_{m}} b_{i} b_{j}\right)-3 a b_{i} b_{j} b_{m}\right]-2 b^{2}\left(a_{i j} b_{m}+a_{i m} b_{j}+a_{j m} b_{i}\right)}{b^{4}} \ln \left(\frac{v+b D_{1}}{v+b D_{2}}\right)
\end{aligned}
$$

Where

$$
\begin{aligned}
\frac{\partial a}{\partial n_{i}} & =2 \sum_{j=1}^{c} n_{j} a_{i j} \\
\delta_{i j m} & =\left\{\begin{array}{l}
1(i=j=m) \\
0(i \neq j, j \neq k \text { or } i \neq m)
\end{array}\right.
\end{aligned}
$$

$$
\delta_{i j}=\left\{\begin{array}{l}
1(i=j) \\
0(i \neq j)
\end{array}\right.
$$

Considering the parameters defined in the main text of the paper, and reorganizing the same terms in the equations (A3) and (A4), the two equations can be written as Equations (3) and (4). 